\title{
Transverse to longitudinal emittance exchange
}

\author{
M. Cornacchia* and P. Emma ${ }^{\dagger}$ \\ Stanford Linear Accelerator Center, Stanford University, Stanford, California 94309
}

(Received 13 June 2002; published 29 August 2002)

\begin{abstract}
A scheme is proposed to exchange the transverse and longitudinal emittances of an electron bunch. A general analysis is presented and a specific beam line is used as an example where the emittance exchange is achieved by placing a transverse deflecting mode radio-frequency cavity in a magnetic chicane. In addition to reducing the transverse emittance, the bunch length is also simultaneously compressed. The scheme has the potential to introduce an added flexibility to the control of electron beams and to provide some contingency for the achievement of emittance and peak-current goals in free-electron lasers.
\end{abstract}

DOI: 10.1103/PhysRevSTAB.5.084001

\section{INTRODUCTION}

The main challenge of the Linac Coherent Light Source [1] and other free-electron lasers (FEL) that are currently planned or under design remains the achievement of a bright electron beam in the transverse plane. Although the FEL also requires a small longitudinal emittance, it appears to be more easily obtainable than that of the transverse plane. In fact, the predictions are that the incoherent momentum spread originating from the photoinjector is too small to effectively damp the microbunching instability induced by coherent synchrotron radiation (CSR) in bunch compressors [2-4]. A motivation therefore exists to reduce the transverse emittance and increase the longitudinal, since this may lead to self-amplified spontaneous emission lasing in a shorter undulator length and simultaneously less CSR microbunching in the compressor.

We show that, under certain conditions, a transfer of emittance from the transverse to the longitudinal plane (and the reverse) is possible and not impractical. Our implementation uses a radio-frequency cavity in a dispersive region of a four dipole-magnet chicane. The cavity operates in the dipole mode, having a longitudinal electric field with gradient such that its strength varies linearly with transverse distance from the axis. A time dependent magnetic deflecting field is also present. A complete emittance analysis is presented and a specific example is studied.

\section{THE DIPOLE MODE CAVITY}

Occasionally, an application arises of an rf cavity operating in a dipole mode, where the longitudinal electric field varies linearly with transverse distance from the axis. The earliest mention of such cavities, to the authors' knowledge, appeared in Ref. [5]. The hope of using such cavities to change the damping of the three modes of oscillation of particles in an electron circular accelerator was dashed by Robinson's classic paper [6] that shows that the partition

*Electronic address: Cornacchia@SLAC.Stanford.edu

${ }^{\dagger}$ Electronic address: Emma@SLAC.Stanford.edu
PACS numbers: 29.27.-a

numbers cannot be changed with an rf field. A discussion of the physical mechanism of this general principle as it applies to a dipole mode cavity was presented in Ref. [7]. Cylindrical cavities operating in the $\mathrm{TM}_{210}$ mode (thus with a quadratic dependence of the longitudinal electric field on the distance from the axis) to couple the longitudinal and transverse motion to enhance laser cooling of ions in a storage ring [8], or to establish a correlation between betatron amplitude and momentum deviation to condition an FEL electron beam [9], have also been proposed. For the system under consideration we use a rectangular cavity having a longitudinal electric field which varies linearly with transverse distance, $x$, from the axis, as shown in Fig. 1.

In the neighborhood of the axis $(x \ll a)$ we have an accelerating field for an electron crossing the cavity at time $t$,

$$
E_{z} \approx E_{0} \frac{x}{a} \cos (\omega t), \quad E_{x}=E_{y}=0,
$$
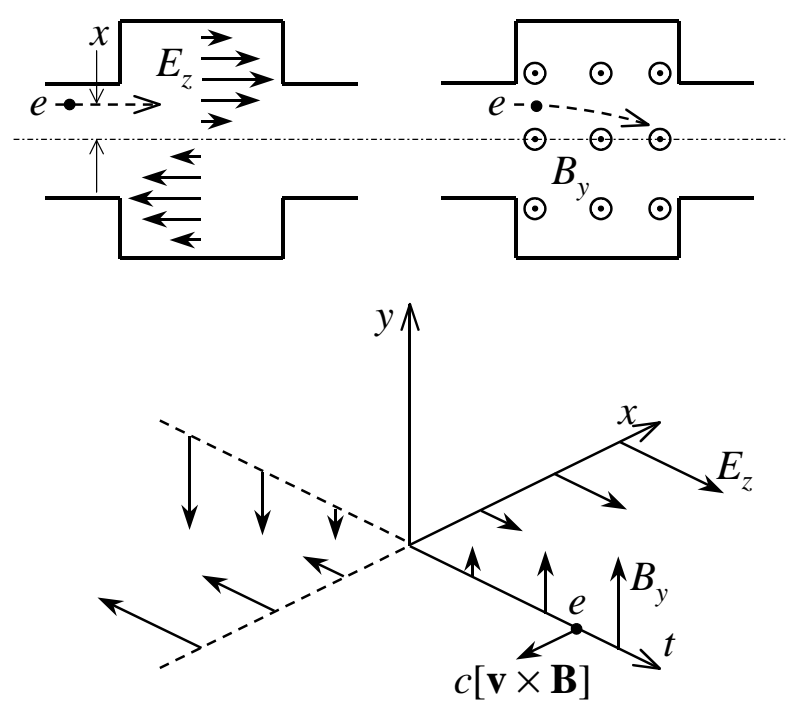

FIG. 1. Electric field (top left) in a dipole mode cavity at synchronous time $(t=0)$, and the magnetic field (top right) one-quarter oscillation later. Longitudinal electric and vertical magnetic fields around $t=0$ (bottom). 
where $z$ is the longitudinal axis of the reference trajectory, $x$ the horizontal axis, $y$ the vertical, $\omega$ the frequency of the cavity oscillations, and $a$ is a constant characteristic of the cavity dimensions. The peak field is $E_{0}=V_{0} / l$, where $V_{0}$ is the peak rf voltage and $l$ the cavity length. The vertical motion is neglected in this analysis, since, to first order, no force of the cavity acts in the vertical plane. The associated magnetic field is obtained from Maxwell's equation:

$\nabla \times \mathbf{E}=-\frac{\partial \mathbf{B}}{\partial t}, \quad B_{y} \approx \frac{E_{0}}{a \omega} \sin (\omega t), \quad B_{x}=B_{z}=0$.

The small relative energy change, $\delta(\equiv \Delta \gamma / \gamma,|\delta| \ll$ 1 ), of an electron traversing the cavity at a distance $x$ from the axis $(|x| \ll a)$ is

$$
\delta \approx \frac{e V_{0}}{E} \frac{x}{a} \cos (\omega t),
$$

where $E$ is the nominal electron energy. We phase the cavity such that the center of the bunch (the reference particle) passes through the cavity at time $t=0$, when the electric field gradient is at its maximum and the magnetic field passes through zero. We consider a bunch length, $\sigma_{z}$, much shorter than the reduced rf wavelength, $\lambda_{\mathrm{rf}} / 2 \pi$ (i.e., $\sigma_{z} \ll \lambda_{\mathrm{rf}} / 2 \pi$ or $\left.|\omega t| \ll 1\right)$. Thus, to first order,

$$
\delta \approx \frac{e V_{0}}{E} \frac{x}{a}=k x, \quad k \equiv \frac{e V_{0}}{a E} .
$$

The horizontal deflection angle due to the vertical magnetic field of the cavity is

$$
\Delta x^{\prime} \approx \frac{e V_{0}}{E} \frac{c t}{a}=k c t \approx k z,
$$

and $z$ is the longitudinal distance from the center of this ultrarelativistic bunch. Note the final choice of dimensions in Sec. V below is consistent with these linear approximations, where $2 \pi \sigma_{z} / \lambda \ll 1$ and $\sigma_{x} / a \ll 1$, and the next order terms in (4) and (5) scale as the cube of these small levels.

\section{EMITTANCE EXCHANGE}

We now analyze the emittance exchange concept and return to the cavity implementation later. The following is a general 4-dimensional linear beam transport analysis [10] in the $x-z$ plane (or $x-y$ plane). The initial uncoupled $4 \times 4$ beam covariance matrix, $\sigma_{0}$, can be written as [11]

$$
\begin{aligned}
\boldsymbol{\sigma}_{0}=\left[\begin{array}{cccc}
\varepsilon_{x_{0}} \beta_{x} & -\varepsilon_{x_{0}} \alpha_{x} & 0 & 0 \\
-\varepsilon_{x_{0}} \alpha_{x} & \varepsilon_{x_{0}} \gamma_{x} & 0 & 0 \\
0 & 0 & \varepsilon_{z_{0}} \beta_{z} & -\varepsilon_{z_{0}} \alpha_{z} \\
0 & 0 & -\varepsilon_{z_{0}} \alpha_{z} & \varepsilon_{z_{0}} \gamma_{z}
\end{array}\right] \\
=\left[\begin{array}{cc}
\boldsymbol{\sigma}_{x} & \mathbf{0} \\
\mathbf{0} & \boldsymbol{\sigma}_{z}
\end{array}\right],
\end{aligned}
$$

where $\alpha_{x, z}, \beta_{x, z}$, and $\gamma_{x, z}\left(\equiv\left\{1+\alpha_{x, z}^{2}\right\} / \beta_{x, z}\right)$ are the beam envelope functions, and $\epsilon_{x_{0}}$ and $\epsilon_{z_{0}}$ are the initial uncoupled beam emittances in the horizontal and longitudinal planes. The rms beam sizes (horizontal and longitudinal) are related to the respective rms emittances by

$$
\sigma_{x}=\sqrt{\varepsilon_{x_{0}} \beta_{x}}, \quad \sigma_{z}=\sqrt{\varepsilon_{z_{0}} \beta_{z}} .
$$

The bunch "chirp," or linear energy slope along the bunch length, is related to the longitudinal parameters by

$$
\frac{\langle z \delta\rangle}{\sigma_{z}^{2}}=-\frac{\alpha_{z}}{\beta_{z}},
$$

with the total rms relative energy spread, $\sigma_{\delta}$, given by

$$
\sigma_{\delta}=\sqrt{\varepsilon_{z_{0}}\left(1+\alpha_{z}^{2}\right) / \beta_{z}}=\sqrt{\sigma_{\delta_{u}}^{2}+\sigma_{\delta_{c}}^{2}} .
$$

Here $\sigma_{\delta_{u}}$ and $\sigma_{\delta_{c}}$ are the time-uncorrelated and timecorrelated relative energy spread components, respectively, which add in quadrature. The normalized longitudinal emittance is

$$
\gamma \varepsilon_{z}=\gamma \sqrt{\sigma_{z}^{2} \sigma_{\delta}^{2}-\langle z \delta\rangle^{2}},
$$

with $\gamma\left(=E / m c^{2}\right)$ the beam energy in units of electron rest mass. In the simple case, with no time-correlated energy spread (i.e., $\langle z \delta\rangle=0$ ), the longitudinal emittance is

$$
\gamma \varepsilon_{z}\left(\alpha_{z}=0\right)=\gamma \sigma_{z} \sigma_{\delta}=\sigma_{z} \frac{\sigma_{E}}{m c^{2}},
$$

where $\sigma_{E}$ is the absolute rms energy spread.

Now propagate the beam through a $4 \times 4$ beam line transfer matrix, $\mathbf{R}$, starting from an initial beam $\boldsymbol{\sigma}_{0}$, with $\mathbf{R}^{T}$ as the transpose of the matrix $\mathbf{R}$.

$$
\boldsymbol{\sigma}=\mathbf{R} \boldsymbol{\sigma}_{0} \mathbf{R}^{T}
$$

Since $\mathbf{R}$ is symplectic and therefore $\operatorname{det}(\mathbf{R}) \equiv|\mathbf{R}|=1$, the $4 \mathrm{D}$ emittance $\left(=\epsilon_{x_{0}} \epsilon_{z_{0}}\right.$ ) of $\boldsymbol{\sigma}_{0}$ is unchanged by $\mathbf{R}$. The $4 \times 4$ matrix $\mathbf{R}$ is constructed from four $2 \times 2$ blocks, $\mathbf{A}$, $\mathbf{B}, \mathbf{C}$, and $\mathbf{D}$ (as in Ref. [12]), as

$$
\mathbf{R}=\left[\begin{array}{ll}
\mathbf{A} & \mathbf{B} \\
\mathbf{C} & \mathbf{D}
\end{array}\right]
$$

with

$$
\mathbf{A}=\left(\begin{array}{ll}
a_{11} & a_{12} \\
a_{21} & a_{22}
\end{array}\right), \quad \mathbf{B}=\left(\begin{array}{ll}
b_{11} & b_{12} \\
b_{21} & b_{22}
\end{array}\right), \text { etc. }
$$

and it follows from (12) that the propagated beam, after beam line $\mathbf{R}$, is

$$
\boldsymbol{\sigma}=\left(\begin{array}{cc}
\mathbf{A} \boldsymbol{\sigma}_{x} \mathbf{A}^{T}+\mathbf{B} \boldsymbol{\sigma}_{z} \mathbf{B}^{T} & \mathbf{A} \boldsymbol{\sigma}_{x} \mathbf{C}^{T}+\mathbf{B} \boldsymbol{\sigma}_{z} \mathbf{D}^{T} \\
\mathbf{C} \boldsymbol{\sigma}_{x} \mathbf{A}^{T}+\mathbf{D} \boldsymbol{\sigma}_{z} \mathbf{B}^{T} & \mathbf{C} \boldsymbol{\sigma}_{x} \mathbf{C}^{T}+\mathbf{D} \boldsymbol{\sigma}_{z} \mathbf{D}^{T}
\end{array}\right),
$$

with $\boldsymbol{\sigma}_{x}$ and $\boldsymbol{\sigma}_{z}$ the initial $2 \times 2$ block matrices of the $x$ and $z$ planes as shown in (6). The squares of the projected rms $x$ and $z$ emittances are the determinants of the $2 \times 2$ on-diagonal blocks, 


$$
\begin{aligned}
\varepsilon_{x}^{2} & =\left|\mathbf{A} \boldsymbol{\sigma}_{x} \mathbf{A}^{T}+\mathbf{B} \boldsymbol{\sigma}_{z} \mathbf{B}^{T}\right| \\
\varepsilon_{z}^{2} & =\left|\mathbf{C} \boldsymbol{\sigma}_{x} \mathbf{C}^{T}+\mathbf{D} \boldsymbol{\sigma}_{z} \mathbf{D}^{T}\right| .
\end{aligned}
$$

We recall that the determinant of the sum of $2 \times 2$ matrices can be expressed using the trace (tr) as

$$
|\mathbf{X}+\mathbf{Y}|=|\mathbf{X}|+|\mathbf{Y}|+\operatorname{tr}\left\{\mathbf{X}^{a} \mathbf{Y}\right\},
$$

where $\mathbf{X}^{a}$ is the adjoint (or symplectic conjugate) of $\mathbf{X}$ (used here to avoid inverting $\mathbf{A}, \mathbf{B}, \mathbf{C}$, or $\mathbf{D}$, which may be singular).

$$
\begin{gathered}
\mathbf{X}^{a}=|\mathbf{X}| \mathbf{X}^{-1}, \mathbf{X} \mid \neq 0, \quad \text { or } \quad \mathbf{X}^{a}=\mathbf{J}^{-1} \mathbf{X}^{T} \mathbf{J} \\
\text { with } \mathbf{J} \equiv\left(\begin{array}{cc}
0 & 1 \\
-1 & 0
\end{array}\right), \mathbf{J}^{2}=-\mathbf{I} .
\end{gathered}
$$

Applying (17), (16) becomes

$$
\begin{aligned}
& \varepsilon_{x}^{2}=|\mathbf{A}|^{2} \varepsilon_{x_{0}}^{2}+|\mathbf{B}|^{2} \varepsilon_{z_{0}}^{2}+\operatorname{tr}\left\{\left(\mathbf{A} \boldsymbol{\sigma}_{x} \mathbf{A}^{T}\right)^{a} \mathbf{B} \boldsymbol{\sigma}_{z} \mathbf{B}^{T}\right\}, \\
& \varepsilon_{z}^{2}=|\mathbf{C}|^{2} \varepsilon_{x_{0}}^{2}+|\mathbf{D}|^{2} \varepsilon_{z_{0}}^{2}+\operatorname{tr}\left\{\left(\mathbf{C} \boldsymbol{\sigma}_{x} \mathbf{C}^{T}\right)^{a} \mathbf{D} \boldsymbol{\sigma}_{z} \mathbf{D}^{T}\right\},
\end{aligned}
$$

where $|\mathbf{A}|,|\mathbf{B}|,|\mathbf{C}|$, and $|\mathbf{D}|$, are the determinants of the $2 \times 2$ blocks of the transfer matrix $\mathbf{R}$. Using an alternate form for the initial uncoupled beam, $\boldsymbol{\sigma}_{x}$ and $\boldsymbol{\sigma}_{z}$,

$$
\begin{array}{ll}
\boldsymbol{\sigma}_{x}=\varepsilon_{x_{0}} \mathbf{Q}_{x} \mathbf{Q}_{x}^{T}, & \mathbf{Q}_{x} \equiv \frac{1}{\sqrt{\beta_{x}}}\left(\begin{array}{cc}
\beta_{x} & 0 \\
-\alpha_{x} & 1
\end{array}\right), \\
\boldsymbol{\sigma}_{z}=\varepsilon_{z_{0}} \mathbf{Q}_{z} \mathbf{Q}_{z}^{T}, & \mathbf{Q}_{z} \equiv \frac{1}{\sqrt{\beta_{z}}}\left(\begin{array}{cc}
\beta_{z} & 0 \\
-\alpha_{z} & 1
\end{array}\right),
\end{array}
$$

and the property of the trace: $\operatorname{tr}\{\mathbf{X Y Z}\}=\operatorname{tr}\{\mathbf{Y Z X}\}=$ $\operatorname{tr}\{\mathbf{Z X Y}\}$, we obtain, from (19)

$$
\begin{aligned}
\varepsilon_{x}^{2} & =|\mathbf{A}|^{2} \varepsilon_{x_{0}}^{2}+|\mathbf{B}|^{2} \varepsilon_{z_{0}}^{2}+\varepsilon_{x_{0}} \varepsilon_{z_{0}} \operatorname{tr}\left\{\mathbf{U} \mathbf{U}^{T}\right\}, \\
\varepsilon_{z}^{2} & =|\mathbf{C}|^{2} \varepsilon_{x_{0}}^{2}+|\mathbf{D}|^{2} \varepsilon_{z_{0}}^{2}+\varepsilon_{x_{0}} \varepsilon_{z_{0}} \operatorname{tr}\left\{\mathbf{V} \mathbf{V}^{T}\right\},
\end{aligned}
$$

where

$$
\begin{aligned}
\mathbf{U} & \equiv \mathbf{Q}_{x}^{-1} \mathbf{A}^{a} \mathbf{B} \mathbf{Q}_{z}, \\
\mathbf{V} & \equiv \mathbf{Q}_{x}^{-1} \mathbf{C}^{a} \mathbf{D} \mathbf{Q}_{z} .
\end{aligned}
$$

The following symplectic relations between submatrix determinants are then used to simplify (21). These relationships are derived in detail in Appendix A (see also Ref. [12]).

$$
|\mathbf{A}|+|\mathbf{C}|=1, \quad|\mathbf{A}|=|\mathbf{D}|, \quad|\mathbf{B}|=|\mathbf{C}| .
$$
by

The $\mathbf{U}$ and $\mathbf{V}$ matrices of (22) are shown to be related

$$
\mathbf{V}=\mathbf{Q}_{x}^{-1} \mathbf{C}^{a} \mathbf{D} \mathbf{Q}_{z}=\mathbf{Q}_{x}^{-1}\left(\mathbf{J}^{-1} \mathbf{C}^{T} \mathbf{J}\right) \mathbf{D} \mathbf{Q}_{z},
$$

using (18), and from the upper right off-diagonal $2 \times 2$ block of (A2), $\mathbf{C}^{T} \mathbf{J D}=-\mathbf{A}^{T} \mathbf{J B}$, so that

$$
\begin{aligned}
\mathbf{V} & =\mathbf{Q}_{x}^{-1} \mathbf{J}^{-1}\left(\mathbf{C}^{T} \mathbf{J D}\right) \mathbf{Q}_{z}=\mathbf{Q}_{x}^{-1} \mathbf{J}^{-1}\left(-\mathbf{A}^{T} \mathbf{J B}\right) \mathbf{Q}_{z} \\
& =-\mathbf{Q}_{x}^{-1} \mathbf{A}^{a} \mathbf{B} \mathbf{Q}_{z}=-\mathbf{U},
\end{aligned}
$$

and therefore

$$
\operatorname{tr}\left\{\mathbf{U} \mathbf{U}^{T}\right\}=\operatorname{tr}\left\{\mathbf{V} \mathbf{V}^{T}\right\}
$$

This trace is the magnitude of the coupling and is the sum of the squares of the four elements of the normalized coupling block of the transfer matrix (i.e., $\mathbf{U}$ or $\mathbf{V}$ ) and is positive. That is,

$$
\operatorname{tr}\left\{\mathbf{U} \mathbf{U}^{T}\right\}=U_{11}^{2}+U_{12}^{2}+U_{21}^{2}+U_{22}^{2} \equiv \lambda^{2} \geq 0 .
$$

The emittances at the exit of the beam line in (21) are now related to the emittances at the start of the beam line (subscript "0"), using (23), (26), and the definition of $\lambda^{2}$ in (27), by

$$
\begin{aligned}
& \varepsilon_{x}^{2}=|\mathbf{A}|^{2} \varepsilon_{x_{0}}^{2}+(1-|\mathbf{A}|)^{2} \varepsilon_{z_{0}}^{2}+\varepsilon_{x_{0}} \varepsilon_{z_{0}} \lambda^{2}, \\
& \varepsilon_{z}^{2}=(1-|\mathbf{A}|)^{2} \varepsilon_{x_{0}}^{2}+|\mathbf{A}|^{2} \varepsilon_{z_{0}}^{2}+\varepsilon_{x_{0}} \varepsilon_{z_{0}} \lambda^{2} .
\end{aligned}
$$

From (28), if $\varepsilon_{x_{0}}=\varepsilon_{z_{0}}$, then $\varepsilon_{x}=\varepsilon_{z}$. That is, equal initial uncoupled emittances will always remain equal through a symplectic map. Similarly, equal uncoupled emittances cannot be generated from unequal uncoupled initial emittances. (Setting $|\mathbf{A}|=\frac{1}{2}$ produces equal emittances, but they are then highly coupled with $\lambda^{2} \neq 0$.) Additionally, if $\lambda^{2}$ is insignificant, which it can be, then setting $|\mathbf{A}|=0$ will produce a complete $x$ - to $z$-plane emittance exchange. Note that $\lambda^{2}=0$ if and only if all $A_{i j}=0$, or the trivial case of no coupling at all, where all $B_{i j}=C_{i j}=0$.

\section{AN EMITTANCE EXCHANGER BEAM LINE}

We then apply this derivation to the chicane and dipole mode cavity system shown in Fig. 2. A magnetic chicane sets up a dispersive region at its center, where the cavity is located. The chicane, of full length $L$, is made of four bending magnets and no quadrupole magnets.

From (4) and (5), the transfer matrix of the "thin-lens" cavity is

$$
\mathbf{R}_{k}=\left[\begin{array}{cccc}
1 & 0 & 0 & 0 \\
0 & 1 & k & 0 \\
0 & 0 & 1 & 0 \\
k & 0 & 0 & 1
\end{array}\right],
$$

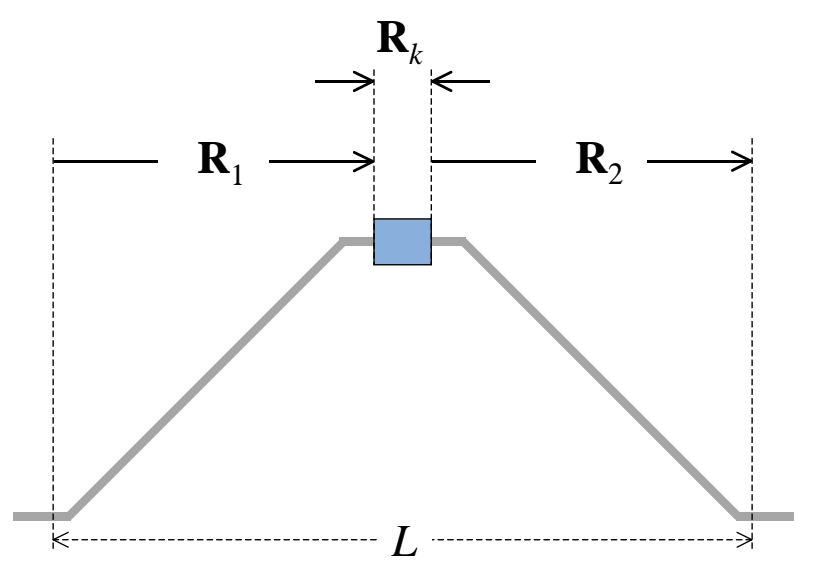

FIG. 2. (Color) Schematic diagram of the chicane and transverse cavity at its center. 
which is similar to a thin-lens skew quadrupole transfer matrix, but in $\left(x, x^{\prime}, z, \delta\right)$ space, rather than $\left(x, x^{\prime}\right.$, $\left.y, y^{\prime}\right)$ space. (The effects of a thick lens are discussed in Sec. VI). The transfer matrix across the entire chicane is

$$
\mathbf{R}=\mathbf{R}_{2} \mathbf{R}_{k} \mathbf{R}_{1},
$$

where $\mathbf{R}_{1}$ and $\mathbf{R}_{2}$ are the transfer matrices of the first and second sections of the chicane, respectively (see Fig. 2), and $\xi$ is the momentum compaction $\left(\xi \equiv R_{56}\right)$ of the full chicane $(\xi>0$ for chosen coordinates with bunch head at $z>0)$.

$$
\begin{aligned}
\mathbf{R}_{1}= & {\left[\begin{array}{cccc}
1 & L / 2 & 0 & \eta \\
0 & 1 & 0 & 0 \\
0 & \eta & 1 & \xi / 2 \\
0 & 0 & 0 & 1
\end{array}\right], } \\
\mathbf{R}_{2}= & {\left[\begin{array}{cccc}
1 & L / 2 & 0 & -\eta \\
0 & 1 & 0 & 0 \\
0 & -\eta & 1 & \xi / 2 \\
0 & 0 & 0 & 1
\end{array}\right] . }
\end{aligned}
$$

The matrix of the full chicane and cavity system is

$$
\mathbf{R}=\left[\begin{array}{cccc}
1-\eta k & L & k L / 2 & k\left(\frac{\xi L}{4}-\eta^{2}\right) \\
0 & 1+\eta k & k & k \xi / 2 \\
k \xi / 2 & k\left(\frac{\xi L}{4}-\eta^{2}\right) & 1-\eta k & \xi \\
k & k L / 2 & 0 & 1+\eta k
\end{array}\right]
$$

$$
|\mathbf{A}|=|\mathbf{D}|=1-\eta^{2} k^{2}, \quad|\mathbf{B}|=|\mathbf{C}|=\eta^{2} k^{2} .
$$

The expression for $\lambda^{2}$ has four terms and is quite long and awkward, even for this system.

$$
\begin{gathered}
\mathbf{U}=\mathbf{Q}_{x}^{-1} \mathbf{A}^{a} \mathbf{B} \mathbf{Q}_{z}^{-1}, \\
\lambda^{2}=\operatorname{tr}\left\{\mathbf{U} \mathbf{U}^{T}\right\} \Rightarrow 4 \text { terms. }
\end{gathered}
$$

A simpler form of $\lambda^{2}$ is easily written by assuming $\eta k=$ 1 (i.e., $|\mathbf{A}|=0$ ).

$$
\lambda^{2}=\frac{4\left(1+\alpha_{x}^{2}\right)\left(1+\alpha_{z}^{2}\right)}{k^{2} \beta_{x} \beta_{z}}=\frac{4 \sigma_{x^{\prime}}^{2} \sigma_{\delta}^{2} \eta^{2}}{\varepsilon_{x_{0}} \varepsilon_{z_{0}}} .
$$

Thus the emittances at the end of the chicane can be exchanged up to a cross term which is related to the rms divergence, $\sigma_{x^{\prime}}$, and energy spread, $\sigma_{\delta}$, of the initial beam, or

$$
\begin{aligned}
\varepsilon_{x} & =\varepsilon_{z_{0}} \sqrt{1+\frac{4\left(1+\alpha_{x}^{2}\right)\left(1+\alpha_{z}^{2}\right)}{k^{2} \beta_{x} \beta_{z}}\left(\frac{\varepsilon_{x_{0}}}{\varepsilon_{z_{0}}}\right)} \\
& =\sqrt{\varepsilon_{z_{0}}^{2}+4 \sigma_{x^{\prime}}^{2} \sigma_{\delta}^{2} \eta^{2}}>\varepsilon_{z_{0}}, \\
\varepsilon_{z} & =\varepsilon_{x_{0}} \sqrt{1+\frac{4\left(1+\alpha_{x}^{2}\right)\left(1+\alpha_{z}^{2}\right)}{k^{2} \beta_{x} \beta_{z}}\left(\frac{\varepsilon_{z_{0}}}{\varepsilon_{x_{0}}}\right)} \\
& =\sqrt{\varepsilon_{x_{0}}^{2}+4 \sigma_{x^{\prime}}^{2} \sigma_{\delta}^{2} \eta^{2}}>\varepsilon_{x_{0}} .
\end{aligned}
$$

It should be recalled that the parameters, $\beta_{x, z}, \alpha_{x, z}, \varepsilon_{x_{0}, z_{0}}$, $\sigma_{x^{\prime}}$, and $\sigma_{\delta}$, all describe the beam at entrance to the chicane. As demonstrated in the example below, the crossterm coefficient, $\lambda^{2}$, can be made insignificantly small for reasonable beam parameters allowing almost complete emittance exchange.

\section{NUMERICAL EXAMPLE}

For an example, we take for the four-dipole chicane shown in Fig. 2 with an $X$ band rf deflecting cavity $(\omega / 2 \pi \approx 11.4 \mathrm{GHz}, a \approx 1.3 \mathrm{~cm})$ at its center. The beam line and beam parameters at the start of the chicane are listed in Table I.

Here we use $\varepsilon_{z_{0}}<\varepsilon_{x_{0}}$, which is a required condition for the reduction of the transverse emittance, and one which may not be trivially realized. Note the parameters are consistent with the linear field approximations of (4) and (5) with $2 \pi \sigma_{z} / \lambda<0.025$ and $\sigma_{x} / a<0.02$. The very small energy spread at chicane entrance means the horizontal rms beam size in the cavity $\left(\sigma_{x} \approx 240 \mu \mathrm{m}\right)$ is dominated by emittance, is not significantly dispersed, and is much smaller than the cavity dimension $a \approx 1.3 \mathrm{~cm}$.

With these parameters used in (36), we have

$$
\begin{aligned}
& \gamma \varepsilon_{x_{0}}=5 \mu \mathrm{m} \rightarrow \gamma \varepsilon_{x}=\gamma \varepsilon_{z_{0}} \sqrt{1+0.026} \approx 1 \mu \mathrm{m}, \\
& \gamma \varepsilon_{z_{0}}=1 \mu \mathrm{m} \rightarrow \gamma \varepsilon_{z}=\gamma \varepsilon_{x_{0}} \sqrt{1+0.001} \approx 5 \mu \mathrm{m},
\end{aligned}
$$

and have completely exchanged the emittance levels. These results are verified with the computer tracking code TURTLE [13] up to second order. The tracking output is shown in Fig. 3.

The system described here, with $k=1 / \eta$, leaves the $x$ and $z$ planes insignificantly correlated (i.e., $\langle\boldsymbol{x} \boldsymbol{z}\rangle \approx 0$, $\left.\langle x \delta\rangle \approx 0,\left\langle x^{\prime} z\right\rangle \approx 0,\left\langle x^{\prime} \delta\right\rangle \approx 0\right)$. Note that the bunch length is also compressed by a factor of 5 at the chicane exit $\left(\sigma_{z}=100 \mu \mathrm{m} \rightarrow 19 \mu \mathrm{m}\right)$, which is a very desirable feature for an FEL requiring a high peak current. The final bunch length, $\sigma_{z_{f}}$, and energy spread, $\sigma_{\delta_{f}}$, for $k=1 / \eta$, and $\alpha_{x}=\alpha_{z}=0$ are 
TABLE I. Beam and system parameters as an example for emittance exchange.

\begin{tabular}{lccc}
\hline \hline \multicolumn{1}{c}{ Parameter } & Symbol & Value & Unit \\
\hline Initial horizontal normalized emittance & $\gamma \varepsilon_{x_{0}}$ & 5 & $\mu \mathrm{m}$ \\
Initial longitudinal normalized emittance & $\gamma \varepsilon_{z_{0}}$ & 1 & $\mu \mathrm{m}$ \\
Initial horizontal beta function & $\beta_{x}$ & 2.6 & $\mathrm{~m}$ \\
Initial longitudinal beta function & $\beta_{z}$ & 2.9 & $\mathrm{~m}$ \\
Initial horizontal alpha function & $\alpha_{x}$ & 0 & \\
Initial longitudinal alpha function & $\alpha_{z}$ & 0 & \\
Initial rms bunch length & $\sigma_{z}$ & 100 & $\mu \mathrm{m}$ \\
Initial rms relative energy spread & $\sigma_{\delta}$ & 3.4 & $10^{-5}$ \\
Electron energy & $E$ & 150 & $\mathrm{MeV}$ \\
Momentum compaction of chicane $\left(R_{56}\right)$ & $\xi$ & 17.7 & $\mathrm{~mm}$ \\
Full chicane length (with 20-cm long drift & $L$ & 2.8 & $\mathrm{~m}$ \\
$\quad$ either side of thin cavity) & & & \\
Bend angle of each chicane dipole & $|\theta|$ & 5.2 & $\mathrm{deg}$ \\
Horizontal dispersion in center of chicane & $\eta$ & 100 & $\mathrm{~mm}$ \\
Transverse cavity strength parameter & $k$ & 10 & $\mathrm{~m}^{-1}$ \\
Peak rf voltage on crest phase & $V_{0}$ & 20 & $\mathrm{MV}$ \\
Cavity dimension & $a$ & 1.3 & $\mathrm{~cm}$ \\
Cavity rf frequency & $\omega / 2 \pi$ & 11.4 & $\mathrm{GHz}$ \\
\hline \hline
\end{tabular}

$$
\sigma_{z_{f}}^{2}=k^{2} \varepsilon_{x_{0}}\left[\frac{\xi^{2} \beta_{x}}{4}+\frac{1}{\beta_{x}}\left(\frac{\xi L}{4}-\frac{1}{k^{2}}\right)^{2}\right]+\xi^{2} \sigma_{\delta}^{2},
$$

$$
\sigma_{\delta_{f}}^{2}=k^{2} \varepsilon_{x_{0}}\left[\beta_{x}+\frac{L^{2}}{4 \beta_{x}}\right]+4 \sigma_{\delta}^{2} .
$$

The energy spread has also increased to $0.24 \%$, a level that is sensitive to the choice of $\eta(=1 / k)$ and also $\beta_{x}$ at the chicane entrance. In addition, the final $\beta_{x}$ and $\alpha_{x}$ functions are greatly magnified by the transverse deflecting field (in this case $\beta_{x}=2.6 \mathrm{~m} \rightarrow 580 \mathrm{~m}, a_{x}=0 \rightarrow-410$ - see Fig. 3, lower left plot).

In this example the initial energy-time correlation, $\alpha_{z}$, was set to zero. In fact a reasonable tolerance on this condition is acceptable. If the initial energy spread is $\sim 3$
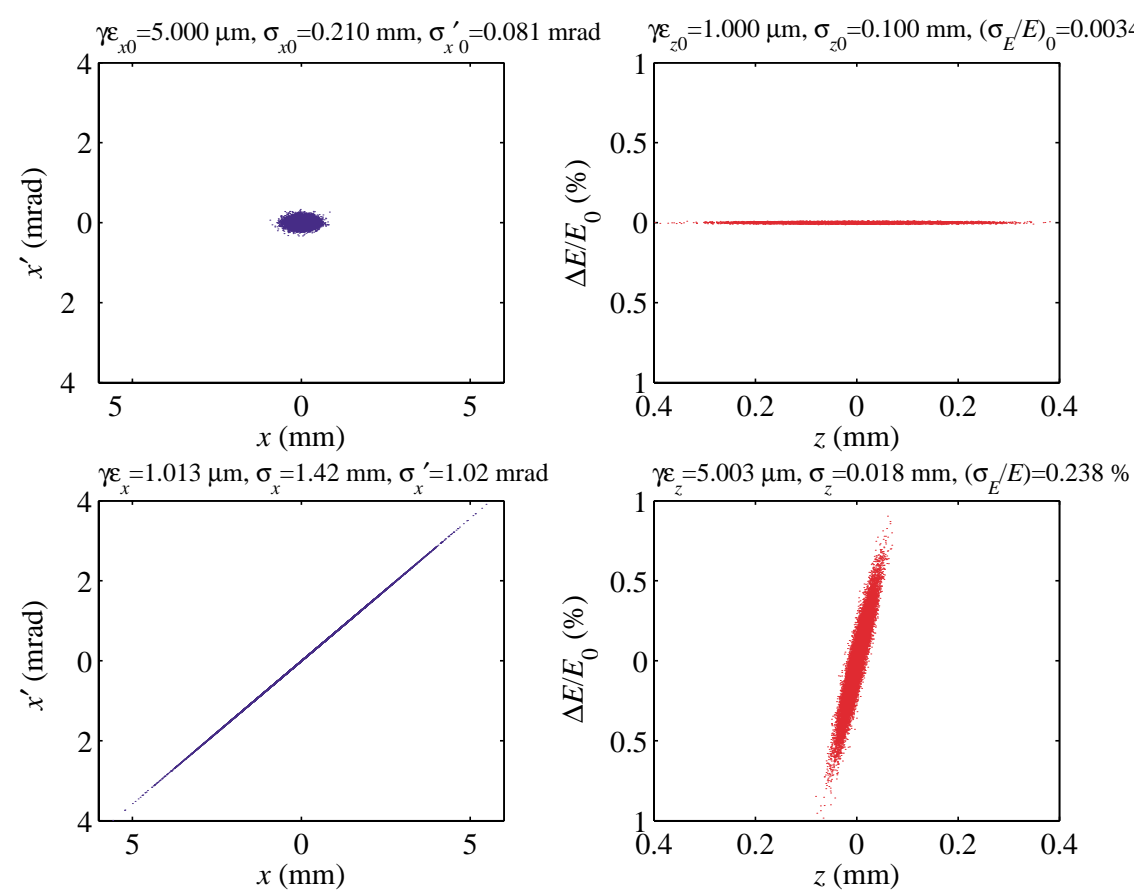

FIG. 3. (Color) Initial (top) and final (bottom) phase space tracking plots. The horizontal and longitudinal emittances are completely exchanged, as predicted by (36). 
times larger due to a linear time correlation $\left[\alpha_{z} \approx 2.8\right.$, see Eq. (8)], the final horizontal emittance is increased by $\sim 10 \%$ in this case, as given by (36). A nonlinear initial energy-time correlation, such as induced by space-charge forces or longitudinal wakefields prior to the chicane, will generate a nonlinear position-angle correlation in transverse phase space after the chicane. The longitudinal and transverse emittances, $\varepsilon_{x_{0}}$ and $\varepsilon_{z_{0}}$, should therefore be considered as projected emittances, which may be increased by nonlinear correlations with their conjugate variables. This presents a practical limitation for the exchange process, where the initial beam may need to be cleaned of aberrations prior to emittance exchange.

Finally, the exchanger beam line has some unusual properties, which may be surprising on first observation. For example, betatron centroid oscillations initiated prior to the chicane will nearly disappear after the chicane (when scaled to local beam size), instead generating energy and timing shifts to the electron bunch $\left(\left\langle x_{0}\right\rangle=1 \sigma_{x} \rightarrow\left\langle z_{f}\right\rangle \approx\right.$ $\left.1 \sigma_{z_{f}}\right)$. On the other hand, bunch arrival time variations upstream of the chicane will not change bunch arrival time after the chicane, instead generating horizontal betatron oscillations. This may be an advantage over standard compressors since it effectively absorbs electron guntiming variations and keeps them from becoming final bunch length and final energy jitter. This behavior is evident in (32) with $\eta k=1$.

\section{THICK-LENS AND SECOND-ORDER EFFECTS}

Second-order optical aberrations can become significant, due mostly to the second-order dispersion from cavity to end of chicane, if the final energy spread (39) becomes too large. This can be controlled by choosing the right initial beta function, $\beta_{x}$, or increasing the chicane dispersion, $\eta$ (which reduces the cavity voltage). The relative emittance increase above the linear calculation of (36), which is due to second-order dispersion, is approximately given by [14]

$$
\frac{\varepsilon_{x_{2}}}{\varepsilon_{x}} \approx \sqrt{1+2\left(\frac{\sigma_{z}}{\eta^{2}} \frac{\varepsilon_{x_{0}}}{\varepsilon_{z_{0}}}\right)^{2}\left(\beta_{x}+\frac{L^{2}}{4 \beta_{x}}\right)^{2}},
$$

where $\sigma_{z}$ and $\beta_{x}$ are the initial beam parameters at the chicane entrance, and $L$ is the full chicane length. In the above case, second-order aberrations are insignificant $\left(\varepsilon_{x_{2}} / \varepsilon_{x} \approx 1.03\right.$ ), but a choice of $\eta=50 \mathrm{~mm}$ (rather than $100 \mathrm{~mm}$ ) and $\beta_{x}=10 \mathrm{~m}$ (rather than $2.6 \mathrm{~m}$ ) causes a factor of 3 final horizontal emittance increase above the linear expectations of (36), and a final rms energy spread of $0.8 \%$ (rather than $0.2 \%$ ). This has been verified using TURTLE tracking. The values for $\beta_{x}$ and $\eta$ should therefore be chosen carefully with (40) as a guide.

The emittance exchanger beam line described above uses a thin-lens model of a transverse deflecting rf cavity to demonstrate the concept. Of course, the cavity will have some length, especially to produce many megavolts. A modification of (29), (32), (35), and (36) is necessary to include this. The symplectic matrix of the thick-lens transverse cavity is

$$
\mathbf{R}_{k}=\left[\begin{array}{cccc}
1 & l & k l / 2 & 0 \\
0 & 1 & k & 0 \\
0 & 0 & 1 & 0 \\
k & k l / 2 & k^{2} l / 6 & 1
\end{array}\right],
$$

where $l$ is the cavity length. Using this in (30), and continuing with the case $\eta k=1$, produces a modified $\mathbf{R}$ with $\mathbf{A}$ and $\mathbf{B}$ blocks which are then used in (34) to calculate $\lambda^{2}$.

$$
\lambda^{2}=\frac{\left(1+\alpha_{x}^{2}\right)\left\{576+48 k^{2} l \xi-4 k^{2} l \alpha_{z} \beta_{z}\left(24+k^{2} l \xi\right)+\alpha_{z}^{2}\left(24+k^{2} l \xi\right)^{2}+k^{4} l^{2}\left(4 \beta_{z}^{2}+\xi^{2}\right)\right\}}{144 k^{2} \beta_{x} \beta_{z}}
$$

With $l=0$ this reduces to (35), but otherwise can be a much more significant limitation in the emittance exchange. If this is now minimized with respect to $\alpha_{z}$, it becomes

$$
\lambda_{\min }^{2}=\frac{4\left(1+\alpha_{x}^{2}\right)\left(1+k^{2} l \xi / 24\right)^{2}}{k^{2} \beta_{x} \beta_{z}},
$$

at a value of $a_{z}$ [related by (8) to the initial energy-time chirp in the bunch], which is given by

$$
\alpha_{z_{\min }}=\frac{1}{12}\left(\frac{k^{2} l \beta_{z}}{1+k^{2} l \xi / 24}\right) \text {. }
$$

The expression for $\lambda^{2}$ in (43) will approach that of (35) with $\alpha_{z}=0$ if $k^{2} l \xi / 24 \ll 1$. For an $X$-band cavity with $\sim 50 \mathrm{MV} / \mathrm{m}$, the level of $20 \mathrm{MV}$ is achieved with $l=0.4 \mathrm{~m}$. From Table I, the values of $k$ and $\xi$ give $k^{2} l \xi / 24 \approx 0.03$. Therefore, the emittance exchanger for a thick lens works almost exactly like the thin lens as long as $\alpha_{z}$ (i.e., the incoming energy chirp) is given by (44) (i.e., $\alpha_{z} \approx 9.4$ in this case). From (9), this means an initial correlated energy spread of $0.03 \%$.

Particle tracking is repeated in Fig. 4 with a thick-lens cavity $(l=0.4 \mathrm{~m})$ and $\alpha_{z}$ set according to (44). In this case, for ease of comparison, the $20-\mathrm{cm}$ long drifts on either side of the cavity in Table I are set to zero to keep the total chicane length constant. The initial energy chirp $\left(\alpha_{z} \neq 0\right)$ is evident in the upper right plot. The emittances are again completely exchanged in this more general and more realistic case. The emittance exchange relations of (36) are now modified for the thick-lens case $(l \neq 0)$, and are given (with $\eta k=1$ ) by 

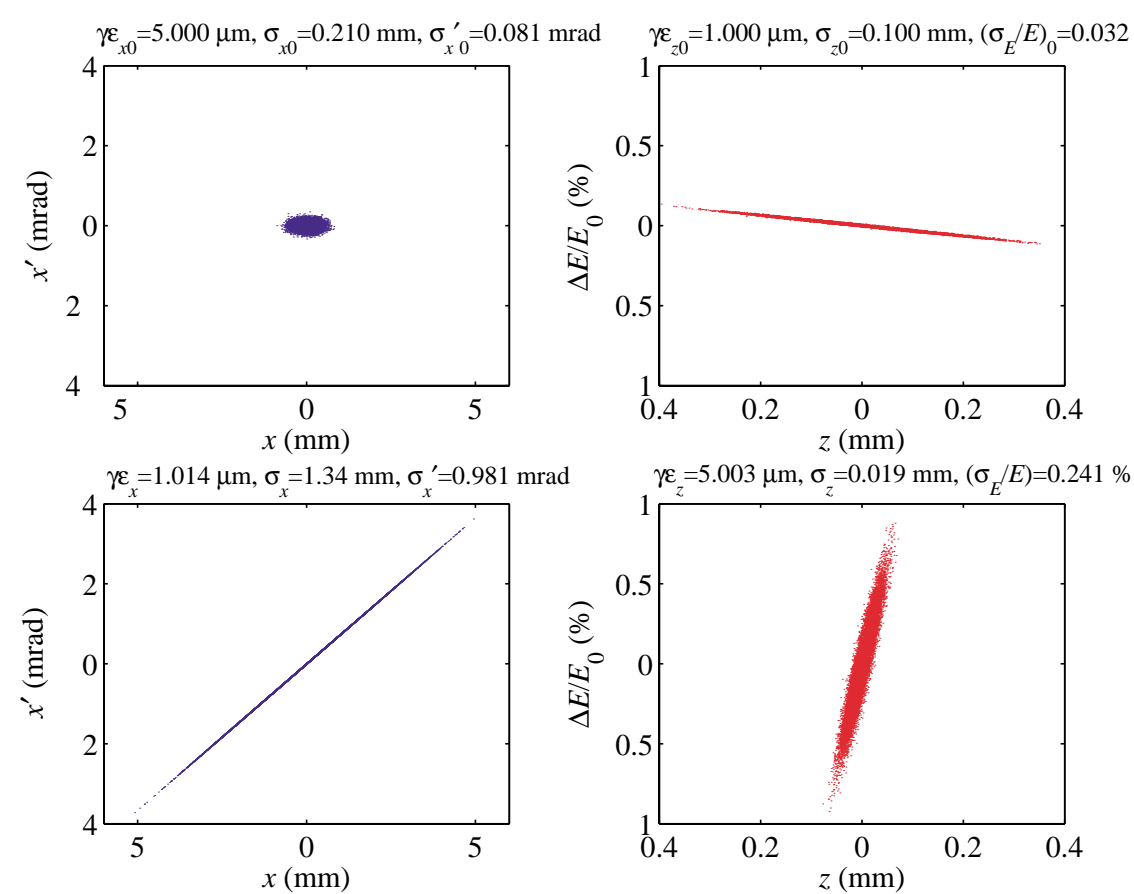

FIG. 4. (Color) Initial (top) and final (bottom) phase space tracking plots with thick-lens cavity and $\alpha_{z}$ set according to (44). The emittances are still completely exchanged.

$\varepsilon_{x}=\varepsilon_{z_{0}} \sqrt{1+\frac{4\left(1+\alpha_{x}^{2}\right)\left(1+k^{2} l \xi / 24\right)^{2}}{k^{2} \beta_{x} \beta_{z}}\left(\frac{\varepsilon_{x_{0}}}{\varepsilon_{z_{0}}}\right)}>\varepsilon_{z_{0}}$,

$\varepsilon_{z}=\varepsilon_{x_{0}} \sqrt{1+\frac{4\left(1+\alpha_{x}^{2}\right)\left(1+k^{2} l \xi / 24\right)^{2}}{k^{2} \beta_{x} \beta_{z}}\left(\frac{\varepsilon_{z_{0}}}{\varepsilon_{x_{0}}}\right)}>\varepsilon_{x_{0}}$.

In (45), the initial energy chirp, $\alpha_{z}$, is not a free parameter and must follow (44). Otherwise (42) must be substituted

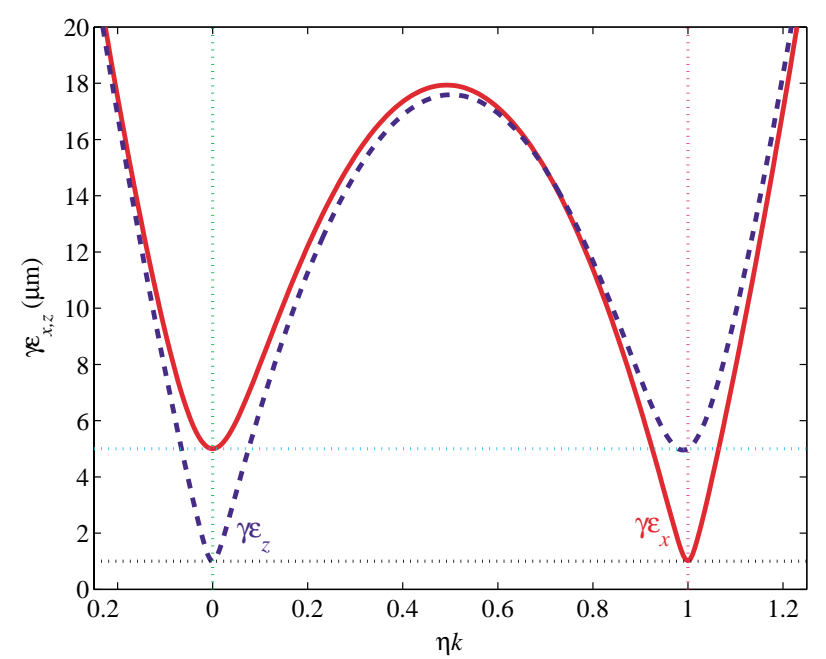

FIG. 5. (Color) Normalized projected emittances (solid/red: $\gamma \varepsilon_{x}$, dashed/blue: $\gamma \varepsilon_{z}$ ) as a function of $\eta k$. Both emittances increase, except at $\eta k=0$ and $\eta k=1$, due to large $x-z$ coupling in the beam. The emittances of the principal planes are evident only at these two points. into (28) for the more general case. Note in (28) that if $\eta k \neq 1$ and $k \neq 0$, both "projected" emittances can simultaneously increase to very large levels, both much larger than the largest initial emittance (see Fig. 5). This is because the beams become highly coupled in this case and the single-plane projected emittances do not reflect the intrinsic beam emittances of the principal planes. The full $4 \mathrm{D}$ phase space volume is, of course, preserved since always $|\mathbf{R}|=1$.

In the particular case described here, the cavity voltage must be set according to $k=1 / \eta$ within $|\Delta k / k|<0.4 \%$ for a final horizontal emittance increase of $<5 \%$, with quadratic sensitivity to the voltage error (see Fig. 5).

\section{APPLICATIONS}

We have shown that, under appropriate conditions, it is possible to transfer the transverse emittance into the longitudinal plane, and the reverse. Reducing both $x$ and $y$ transverse emittances, however, is problematic. The system described here can only exchange emittance values between planes. Since it is symplectic, it cannot arbitrarily vary the emittance ratios and also leave the beams uncoupled. One might imagine two chicane-cavity systems in series, with the second chicane rotated by $90^{\circ}$ in the $x-y$ plane. But since the first chicane raises the $z$ emittance above the transverse goal, the next chicane will swap the $y$ emittance with the new large $z$ emittance, inhibiting round transverse uncoupled beams. Producing small round beams might be accomplished by using this chicanecavity system in conjunction with a flat-beam gun [15], 
which initially produces a sufficiently small vertical emittance.

The advantage of the emittance transfer scheme proposed here is a reduced dependence on the photoinjector to meet the transverse emittance goals, thus adding a considerable safety margin to the design. The chicane also compresses the bunch by altering the path length of the particles with large transverse rf-cavity-induced kicks, thereby adding another useful function to the scheme. The compression takes place entirely in the last bend. Coherent synchrotron radiation or longitudinal wakefields in the first two bends may present a severe limitation if the energy spread is increased significantly. Vacuum chamber shielding or low charge levels may be necessary, depending on bend magnet and beam parameters. An additional bonus is that the reduction of the transverse emittance is accompanied by an increase of the local energy spread, a desirable requisite for the control of the CSR microbunching instability. Finally, the system provides an alternate approach for the manipulation of bunch length, energy spread, and emittance and may add to the flexibility in preparing high-brightness electron beams.

\section{ACKNOWLEDGMENTS}

We would like to thank W. Spence for introducing us to many of the analytical methods employed in this treatment, and $\mathrm{M}$. Woodley for help in running the TURTLE tracking. In addition we thank K. Bane, A. Chao, J. Irwin, A. Kabel, and G. Stupakov for encouragement and useful comments. This work was prepared for the Department of Energy under Contract No. DE-AC03-76SF00515 by Stanford Linear Accelerator Center, Stanford University, Stanford, California, 94309.

\section{APPENDIX A}

To derive the relationships between determinants of the submatrices we start with the symplectic condition $\mathbf{R}^{T} \mathbf{S R}=\mathbf{R S R}^{T}=\mathbf{S}$, with $\mathbf{S}[16]$ as the $4 \times 4$ form of the matrix $\mathbf{J}$, which is defined in (18),

$$
\mathbf{R}^{T} \mathbf{S R}=\mathbf{R S R}^{T}=\mathbf{S}=\left(\begin{array}{ll}
\mathbf{J} & \mathbf{0} \\
\mathbf{0} & \mathbf{J}
\end{array}\right),
$$

or explicitly

$$
\begin{aligned}
\mathbf{R}^{T} \mathbf{S R} & =\left(\begin{array}{ll}
\mathbf{A}^{T} \mathbf{J A}+\mathbf{C}^{T} \mathbf{J C} & \mathbf{A}^{T} \mathbf{J B}+\mathbf{C}^{T} \mathbf{J D} \\
\mathbf{B}^{T} \mathbf{J A}+\mathbf{D}^{T} \mathbf{J C} & \mathbf{B}^{T} \mathbf{J B}+\mathbf{D}^{T} \mathbf{J D}
\end{array}\right) \\
& =\left(\begin{array}{ll}
\mathbf{J} & \mathbf{0} \\
\mathbf{0} & \mathbf{J}
\end{array}\right), \\
\mathbf{R S R}^{T} & =\left(\begin{array}{ll}
\mathbf{A J} \mathbf{A}^{T}+\mathbf{B J} \mathbf{B}^{T} & \mathbf{A J C}^{T}+\mathbf{B J D}^{T} \\
\mathbf{C J} \mathbf{J}^{T}+\mathbf{D J B}^{T} & \mathbf{C J C}^{T}+\mathbf{D J D}^{T}
\end{array}\right) \\
& =\left(\begin{array}{ll}
\mathbf{J} & \mathbf{0} \\
\mathbf{0} & \mathbf{J}
\end{array}\right) .
\end{aligned}
$$

Taking determinants of the on-diagonal blocks from above provides relationships between the submatrices.

$$
\begin{aligned}
\left|\mathbf{A}^{T} \mathbf{J A}+\mathbf{C}^{T} \mathbf{J C}\right| & =\left|\mathbf{A} \mathbf{J} \mathbf{A}^{T}+\mathbf{B} \mathbf{J} \mathbf{B}^{T}\right|=|\mathbf{J}|=1, \\
\left|\mathbf{B}^{T} \mathbf{J B}+\mathbf{D}^{T} \mathbf{J D}\right| & =\left|\mathbf{C} \mathbf{J} \mathbf{C}^{T}+\mathbf{D} \mathbf{J} \mathbf{D}^{T}\right|=|\mathbf{J}|=1 .
\end{aligned}
$$

Applying (17) to one of the above equations (e.g., the first one, at upper left) produces

$$
|\mathbf{A}|^{2}+|\mathbf{C}|^{2}+\operatorname{tr}\left\{\left(\mathbf{A}^{T} \mathbf{J A}\right)^{a} \mathbf{C}^{T} \mathbf{J C}\right\}=1,
$$

and using $\mathbf{X}^{T} \mathbf{J}=\mathbf{J} \mathbf{X}^{a}$ and $\mathbf{X}^{a} \mathbf{X}=|\mathbf{X}| \mathbf{I}$ from (18), and also $(\mathbf{X Y Z})^{a}=\mathbf{Z}^{a} \mathbf{Y}^{a} \mathbf{X}^{a}$, gives

$$
\begin{aligned}
|\mathbf{A}|^{2} & +|\mathbf{C}|^{2}+\operatorname{tr}\left\{\left(\mathbf{J} \mathbf{A}^{a} \mathbf{A}\right)^{a} \mathbf{J} \mathbf{C}^{a} \mathbf{C}\right\} \\
& =|\mathbf{A}|^{2}+|\mathbf{C}|^{2}+|\mathbf{A}||\mathbf{C}| \operatorname{tr}\left\{\mathbf{J}^{a} \mathbf{J}\right\}=1 .
\end{aligned}
$$

Finally, with $\mathbf{J}^{a} \mathbf{J}=\mathbf{I}$, we have

$$
|\mathbf{A}|^{2}+|\mathbf{C}|^{2}+2|\mathbf{A}||\mathbf{C}|=(|\mathbf{A}|+|\mathbf{C}|)^{2}=1 .
$$

Repeating steps (A5) through (A7) for each of the remaining three determinants in (A4) produces

$$
\begin{aligned}
|\mathbf{A}|+|\mathbf{C}|=1, & |\mathbf{B}|+|\mathbf{D}|=1, & |\mathbf{A}|+|\mathbf{B}|=1, \\
& |\mathbf{C}|+|\mathbf{D}|=1, & \text { (A8) }
\end{aligned}
$$

and we have the following relations between submatrix determinants:

$$
|\mathbf{A}|+|\mathbf{C}|=1, \quad|\mathbf{A}|=|\mathbf{D}|, \quad|\mathbf{B}|=|\mathbf{C}| .
$$

[1] SLAC Report No. SLAC-R-593, 2002

[2] S. Heifets, S. Krinsky, and G. Stupakov, Phys. Rev. ST Accel. Beams 5, 064401 (2002).

[3] E. L. Saldin, E. A. Schneidmiller, and M. V. Yurkov, Nucl. Instrum. Methods Phys. Res., Sect. A 483, 516 (2002).

[4] Z. Huang and K.-J. Kim, Phys. Rev. ST Accel. Beams 5, 074401 (2002).

[5] H. G. Hereward, CERN Report No. 58-12, 1958.

[6] K. W. Robinson, Phys. Rev. 111, 373 (1958).

[7] M. Cornacchia and A. Hofmann, in Proceedings of the 1995 Particle Accelerator Conference, Dallas, Texas (IEEE, Piscataway, NJ, 1995)

[8] H. Okamoto, A. Sessler, and D. Mohl, Phys. Rev. Lett. 72, 3977-3980 (1994).

[9] A. M. Sessler, D. H. Whittum, and L.H. Yu, Phys. Rev. Lett. 68, 309-312 (1992).

[10] The matrix treatment used in this paper to describe the coupled $x-z$ motion follows closely the unpublished work developed by W. Spence and P. Emma to describe the horizontally vertically coupled motion in the collider arcs of the Stanford Linear Collider (1991).

[11] K. L. Brown, SLAC Report No. SLAC-PUB-2370, 1980.

[12] K.L. Brown and R.V. Servranckx, SLAC Report No. SLAC-PUB-4679, 1989.

[13] DECAY TURTLE: A Computer Program for Simulating Charged Particle Beam Transport Systems, in D. C. Carey et al., SLAC Report No. SLAC-246, 1982. 
[14] P. Emma (unpublished).

[15] R. Brinkmann, Ya. Derbenev, and K. Flottmann, Phys. Rev. ST Accel. Beams 4, 053501 (2001).

[16] Note that $\mathbf{S}$ is written here for a coordinate system with bunch head located at $z>0$. If $z$ is chosen with bunch head at $z<0$, then $-\mathbf{J}$ should be used in the lower right block of $\mathbf{S}$. 